\title{
A CONTAGIOUS PSEUDOLYMPHOMA: PSEUDOLYMPHOMATOID SYPHILIS
}

\section{Introduction and Objectives}

Cutaneous pseudolymphomas are benign reactive lymphocytic proliferations that mimic clinical and histologically cutaneous lymphomas. They are classified as pseudolymphomas of T or B cells. They can be secondary to drug intake (classically phenytoin), insect bites, tattoos, infections (viruses, bacteria, spirochetes and parasites), vaccines, foreign bodies or exogenous agents (contact allergens, ultraviolet radiation).

\section{Results}

A 59-year-old man with a history of hypertension under treatment with hydrochlorothiazide and enalapril, presented with cutaneous lesions on his face and trunk of almost 4 months of evolution, not pruritic or painful, that do not respond to treatment with topical or systemic corticosteroids.

On physical examination multiple papules and erythematous-violaceous plaques were observed in the frontal region, 1 to $2 \mathrm{~cm}$ in size (fig. 1). A maculopapular and erythematous rash on the neck and thorax was observed (fig. 2). No palmoplantar or mucosal involvement was seen. No adenopathies were detected.

A skin biopsy was performed, which showed a dense inflammatory infiltrate with tendency to epidermotropism. In immunohistochemistry, marked positivity was observed for CD20, and lower for CD3.

A cutaneous lymphoma was suspected.

However, treponemal staining in the skin biopsy was positive. Serum VDRL and treponemal IgG and IgM tests were then performed, and confirmed the diagnosis of luetic disease. A diagnosis of secondary pseudolymphomatoid syphilis was made.

Intramuscular benzathine penicillin (2.4 million IU) was then

administered. Rapid clinical improvement was observed, with complete resolution of the skin lesions.

Histology

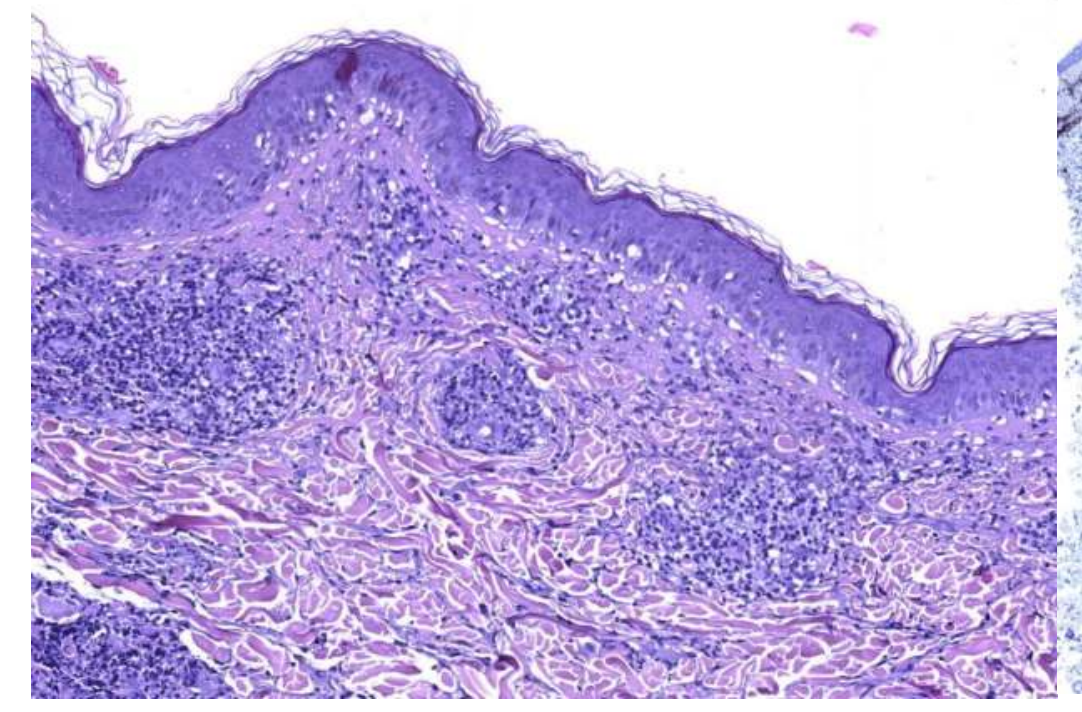

Immunohystochemistry

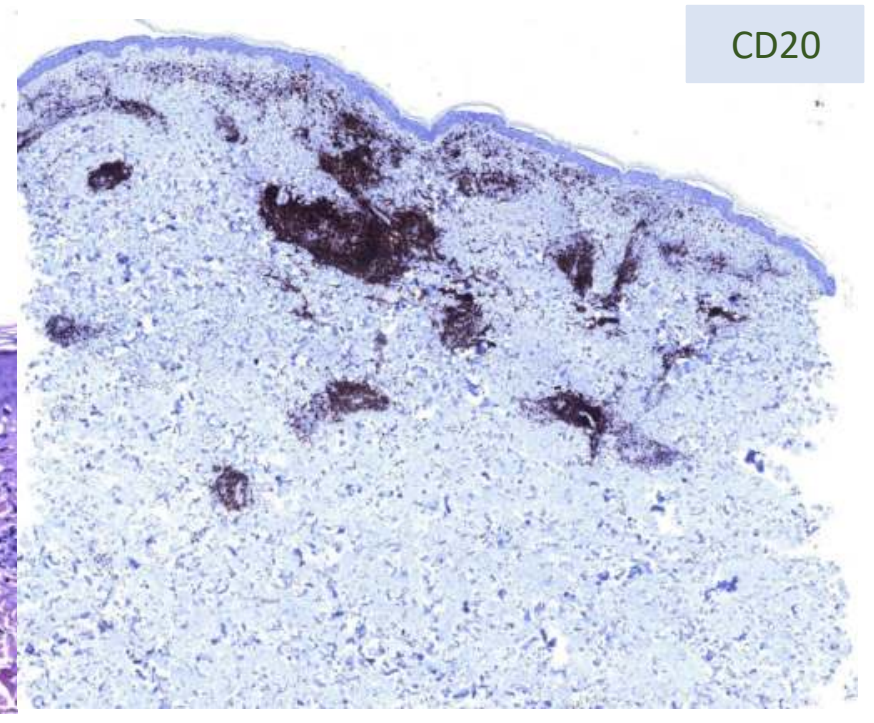

\section{Materials and Methods}

Here we describe a patient who presented a pseudolymphoma as a clinical presentation of a secondary syphilis

Fig. 1

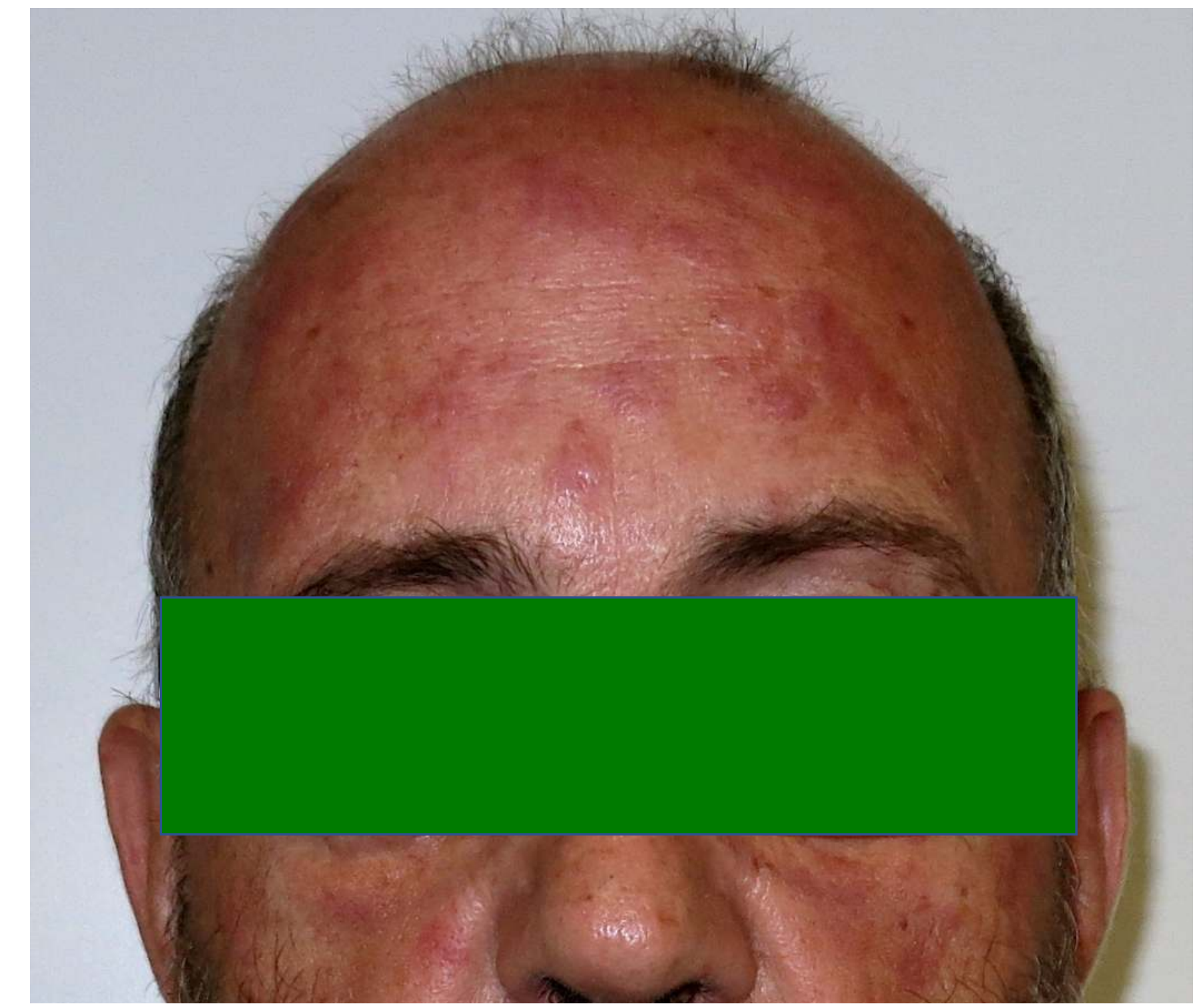

Fig. 2

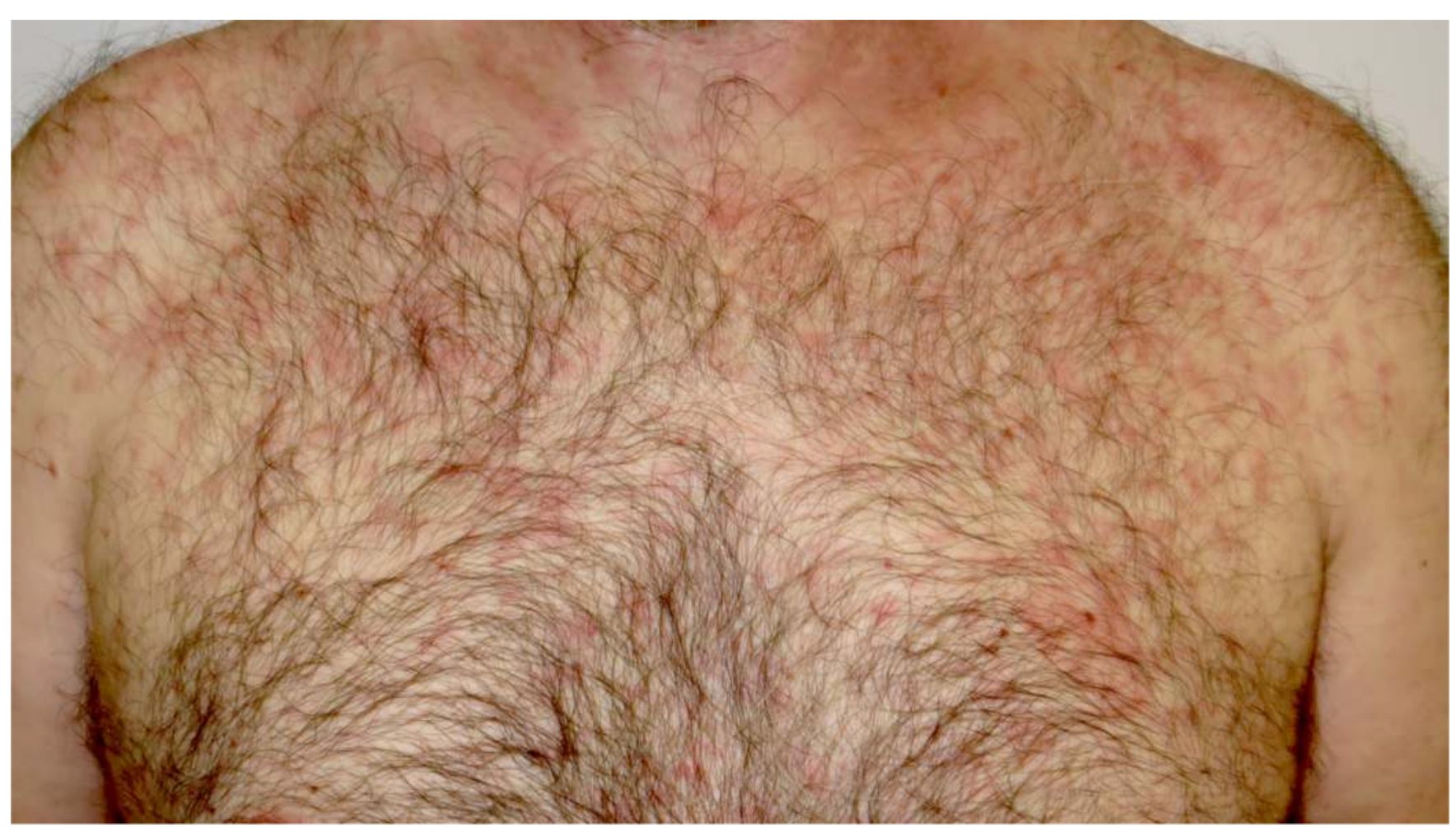

\section{Conclusions}

Secondary syphilis can have various clinical presentations, from classic syphilitic roseola to uncommon forms of presentation such as malignant syphilis and pseudolymphomatoid syphilis. The latter may be indistinguishable from a cutaneous lymphoma. It is essential to have a high index of suspicion and to remember that syphilis is the "great simulator". 